\title{
Generation of single and periodically repeated powerful ultrashort microwave pulses
}

\author{
N.S. Ginzburg, I.V. Zotova \\ Institute of Applied Physics RAS, Nizhny Novgorod, Russia \\ ginzburg@appl.sci-nnov.ru
}

Generation of powerful ultrashort microwave pulses (USP) is of interest for a large number of practical applications, including radars with high resolution, plasma diagnostics, spectroscopy etc. In order to achieve this purpose many methods well-known in laser physics can be implemented in microwave electronics.

In the last decade significant progress was achieved in generation of microwave USPs based on classical superradiance (SR) of extended electron bunches. Using of this effect a new type of pulse sources has been developed, which are capable of producing uniquely short (under $0.2-1 \mathrm{~ns}$ ) electromagnetic pulses at super-high peak powers exceeding $1 \mathrm{GW}$ in the millimeter waves (Kaband) and $3 \mathrm{GW}$ in the centimeter waves (X-band) with a repetition frequency up to several kilohertz [1]. A natural continuation of this research is related with the promotion of SR sources in shorter wavelengths ranges, where the oversized waveguides should be used. In the first part of this paper the results of the experimental observation of SR emission with excitation of a surface wave in the oversized periodically corrugated slow wave structures are presented. The experiments were based on a theoretical consideration in the frame of the quasi-optical approach.

A periodic train of powerful ultrashort microwave pulses with a repetition frequency of 10-100 $\mathrm{MHz}$ can be generated in electron oscillators with a saturable absorber installed in the feedback loop [2]. The mechanism of pulse formation in this case is similar to the passive mode-locking [3] in laser physics where such pulses are also treated as dissipative solitons. The second part of this paper is devoted to the theoretical analysis of solitons generation in a mode-locked two-section microwave oscillator consisting of Cherenkov TWT and a Kompfner absorber in the feedback loop.

\section{Generation of Sub-THz SR Pulses \\ Based on Excitation of Surface Waves in Oversized Waveguides}

Cherenkov SR of electron bunch exciting the surface wave in an oversized corrugated cylindrical waveguide (Fig. 1a) can be considered within a quasi-optical approach [4]. In this case the radiation field near a shallow corrugation is presented as a sum of two counterpropagating $T M$ polarized wave-beams:

$$
H_{\varphi}=\operatorname{Re}\left(A_{+}(z, r, t) e^{i \bar{\omega} t-i k z}+A_{-}(z, r, t) e^{i \bar{\omega} t+i k z}\right),
$$

propagation and mutual coupling of which is described by two non-uniform parabolic equations. The synchronous interaction of electrons with a forward partial waves leads to development a self-bunching and formation of powerful SR pulse.
Simulations show that the most optimal conditions for SR emission correspond to excitation of the backward surface wave near the Bragg frequency ( $\pi$ - regime, Fig.1b). For parameters of an electron bunch formed by an accelerator RADAN (electron energy of $300 \mathrm{keV}$, a total current of $2 \mathrm{kA}$, a bunch duration of $500 \mathrm{ps}$ ) and a corrugated waveguide with the mean radius of $3.75 \mathrm{~mm}$, corrugation period of $0.825 \mathrm{~mm}$, and corrugation depth of $0.36 \mathrm{~mm}$ the operating frequency in the resonant point is of $0.14 \mathrm{THz}\left(2 r_{0} / \lambda \approx 3.5\right)$. In this case the power of generated SR pulse emitted in $-z$ direction achieves $\sim 200 \mathrm{MW}$ for pulse duration of $\sim 200$ ps (Fig. 2a). As it is seen in Fig. $2 b$ the instant spatial structure of the partial wave corresponds to formation of the evanescent surface wave with the field amplitude exponentially decaying from the corrugation.

Based on a theoretical analysis, experiments on observation of the sub-terahertz SR pulse generation were carried out in IEP RAS (Ekaterinburg). Photo of the experimental set-up is shown in Fig. 3. A typical oscilloscope trace of generated SR pulses with a duration of about 150 ps and a rise time of 100 ps reconstructed in the "power-time" coordinates is presented in Fig. 3c. Frequency measurements using a set of cut-off waveguide filters show that the pulse spectrum has a central frequency in the interval $0.13-0.15 \mathrm{THz}$. The peak power of generated SR pulses was estimated by integrating the detector signal over the directional pattern and achieved of 50-70 MW, that strongly exceeds the value obtained in the previous sub-terahertz experiments [5] with singlemode waveguides.

\section{Generation of a Train of Dissipative Solitons in a Cherenkov Oscillator with a Kompfner Absorber in the Feedback Loop}

In microwave electronic the passive mode-locking effect can be realized in a two-section oscillator which consists of an amplifying and an absorbing section Fig. 4a. As an amplifier we consider here a Cherenkov TWT which is of interest for generation of multi-gigawatt microwave pulses. A saturable absorption can be realized also based on Cherenkov TWT operating in the so-called Kompfner dip regime, when the em wave overcomes the electrons [6]. In this case both sections of an USP oscillator are described by following equations:

$$
\begin{aligned}
& \left(\frac{\partial}{\partial z}+s \frac{\partial}{\partial \zeta}\right) \hat{A}=\frac{\omega}{c} \frac{e I_{0} \chi}{m c^{3} \gamma_{0} N} \frac{1}{\pi} \int_{0}^{2 \pi} e^{-i \theta} d \theta_{0}, \\
& \frac{\partial u}{\partial z}=\frac{\omega}{c} \operatorname{Re}\left(\chi \hat{A} e^{i \theta}\right), \quad \frac{\partial \theta}{\partial z}=\frac{\omega}{c}(\Delta+\mu u),
\end{aligned}
$$


where $\zeta=t-z / V_{\| 0}, \hat{A}=e A / m c \omega$ is the normalized field amplitude, $\chi$ is the coupling parameter, $u=\left(1-\gamma / \gamma_{0}\right)$ is the relative energy variation, $\mu>0$ is the inertial grouping parameter, $\Delta=1-V_{\| 0} / V_{p h}$ is the initial resonance detuning, Regime of amplification realizes for $\Delta<0$, while the regime of Kompfner dip - for $\Delta>0$. In accordance with Fig. 4a after passing through the absorber and reflection from the partially transparent mirror, the signal returns to the input of the amplifier with a time delay $T: A(0, \zeta)==R A\left(L_{1}+L_{2}, \zeta-T\right)$, where $R<1$ is the coefficient of reflection.
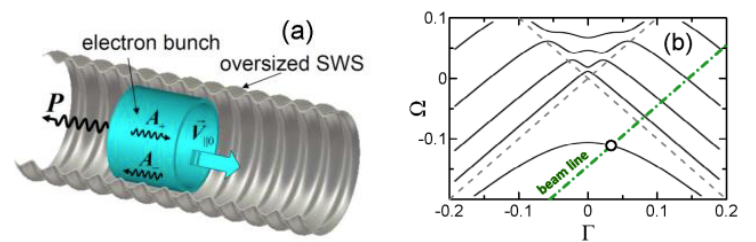

Fig. 1. (a) Scheme of SR pulse generation with excitation of a surface wave in an oversized periodically corrugated waveguide. (b) Dispersion characteristics of a corrugated waveguide and an electron beam

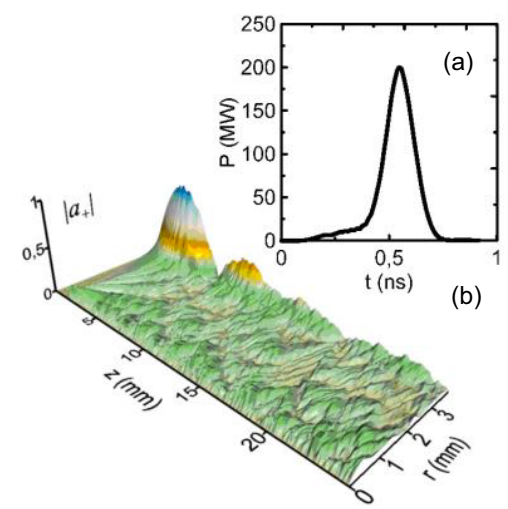

Fig. 2. SR emission with excitation of the backward surface wave: $(a)$ generated SR pulse, $(b)$ the structure of the forward partial wave

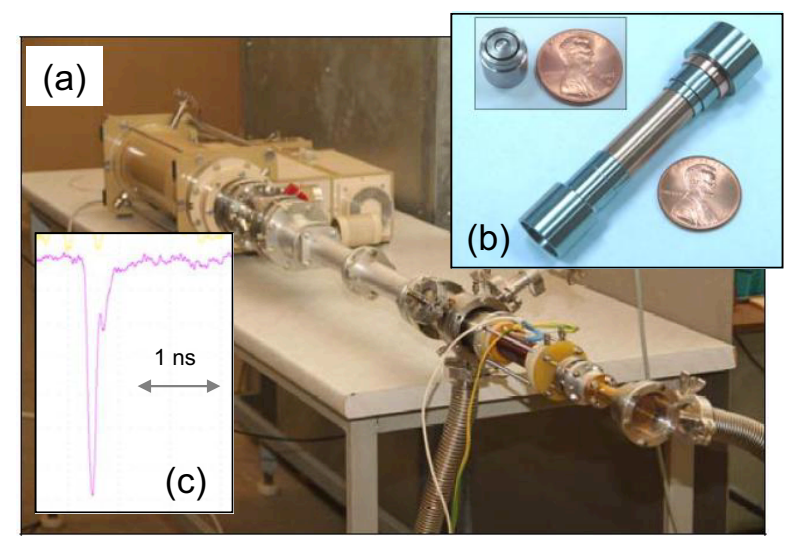

Fig. 3. Photo of the experimental set-up $(a)$, a corrugated waveguide and a coaxial reflector $(b)$ used for observation of superradiance with excitation of a surface wave. (c) Oscilloscope trace of the $0.14 \mathrm{THz}$ SR pulse with duration of $150 \mathrm{ps}$ and peak power up to $70 \mathrm{MW}$

The important factor for effective amplification of ultrashort em pulses is the difference of the slippage pa- rameter $s=V_{g r}^{-1}-V_{\| 0}^{-1}$ from zero. In this case due to slippage of an em pulse over electrons it can effectively accumulates the energy from different beam fractions. On the contrary, in the absorbing section for minimization of the absorber relaxation time the grazing incidence of the electron beam and the em wave is beneficial $s=0$.
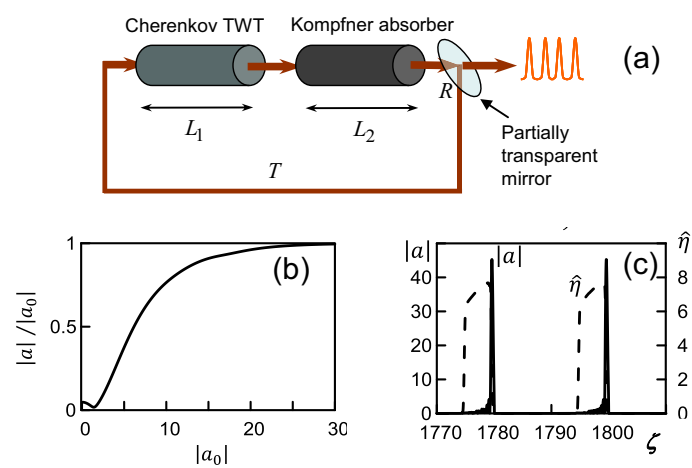

Fig. 4. (a) Scheme of a mode-locked microwave oscillator consisting of a Cherenkov TWT, a Kompfner absorber and a feedback loop. (b) Dependence of a transmission coefficient of the Kompfner absorber on the input signal amplitude demonstrating the saturation of absorption. (c) The output signal and instantaneous efficiency of the USP mode-locked oscillator.

Simulations were performed for following parameters of an $10 \mathrm{GHz}$ amplifier: electron current of $6 \mathrm{kA}$, electron energy of $0.8 \mathrm{MeV}$, the coupling impedance of $2 \mathrm{Ohm}$. In the second section with similar parameters the Kompfner dip with saturation of absorption (Fig. 4b) can be realized by the proper choice of the period and length of a slow wave structure. Installation of such a section in the feedback loop of an electron oscillator leads to realization of passive mode-locking effect and generation of a train of ultrashort $0.8 \mathrm{~ns}$ pulses with peak power up to $6 \mathrm{GW}$. Based on an analogy with laser physics such pulses can be interpreted as dissipative solitons.

This work was supported by Russian Scientific Foundation (RSCF), grant No. 16-42-01078.

\section{References}

1. Korovin, S., Eltchaninov, A., Rostov, A et al. Generation of Cherenkov superradiance pulses with a peak power exceeding the power of driving short electron beam // Phys.Rev.E 2006. V.74 P. 016501

2. Ginzburg, N.S., Denisov, G.G., Vilkov, M.N. et al. Generation of "gigantic" ultra-short microwave pulses based on passive mode-locking effect in electron oscillators with saturable absorber in the feedback loop // Phys. Plasmas. 2016. V. 23. P. 050702-1-4.

3. Haus, H.A. Mode-Locking of Lasers // IEEE J. of Select. Topics in Quant. Electron. 2000. V. 6, No. 6. P. 1173-1185.

4. Ginzburg, N.S., Malkin, A.M., Sergeev, A.S. et al. Generation of Sub-Terahertz Superradiance Pulses Based on Excitation of a Surface Wave by Relativistic Electron Bunches Moving in Oversized Corrugated Waveguides // Phys. Rev. Lett. 2016. V.117 P.204801

5. Yalandin, M.I., Shpak, V.G., Shunailov, S.A., et al Generation of powerful subnanosecond microwave pulses in the range of 38-150 GHz// IEEE Trans. on Plasma Sci. 2000. V.28, Iss. 5 P. 1615

6. Kompfner $R$. On the operation of the traveling wave tube at low level // J. Brit. IRE. 1950. V. 10, No 8-9. P. 283 